PALAVRAS. Revista de Epistemología, Metodología y Ética del Psicoanálisis

ISSN: $2468-9831$

www.revistas.unlp.edu.ar/palavras

palavras@outlook.com.ar

Argentina

\title{
ALGUNAS OBSERVACIONES SOBRE LA CONCEPCIÓN DEL LENGUAJE SUBYACENTE EN EL "PROYECTO DE UNA PSICOLOGÍA PARA NEURÓLOGOS"
}

\author{
DOI 10.24215/24689831e001 \\ Carlos Caorsi
}

Resumen

\begin{abstract}
Freud's theory of language outlined in his Entwurf einer Psychologie, articulates the distinction between two types of representations: thing-presentation and word-presentation. Both types of representations are psychic records of stimulations and originate in sensory perception. But what Freud considered wordpresentation is limited to the material aspects of the sign, as strings of phonemes or graphs, and does not include the meaning that makes this string of phonemes a word. So the word-presentation contains only a part of what we usually consider a word. The other part, its meaning, will be provided by the connection of this word-presentation with the thingpresentation.
\end{abstract}

Acordingly, we have two complexes of sense data, that is, the thing-presentation and the word-presentation, and will be the given connection between them what attributes meaning to the latter. Then the fundamental semantic relationship that will lead to language development will consist of associative connection between two representation complexes. At this point, three issues of fundamental importance that we intend to answer in this paper arise:

1. How is the connection between the wordpresentation and thing-presentation established,

2. How are those complexes decompose to yield an articulate language, and

3. What role does the world thing play, if any.

Key word: Project of Psychology - Thingrepresentation - Word-representation
La teoría del lenguaje que Freud esboza en el Proyecto de una psicología, se articula sobre la distinción entre dos tipos de representaciones: la representación-cosa y la representación-palabra. Ambos tipos de representaciones son registros a nivel psíquico de influjos procedentes de estimulaciones y tienen su origen en la percepción sensorial. Pero lo que Freud considera representación-palabra, se limita a los aspectos materiales del signo, como ristras de fonemas o grafos y no incluye el significado que hace de esa ristra de fonemas una palabra. De modo que la representación-palabra contiene sólo una parte de lo que habitualmente consideramos una palabra. La otra parte, su significado, será provisto por la conexión de la representación-palabra con la representación-cosa. De acuerdo con esto, tenemos dos complejos de sense data, a saber, la representación-cosa y la representación-palabra, y será la conexión que se dé entre ambos lo que atribuirá significado a este último. Luego la relación semántica fundamental que dará lugar al desarrollo del lenguaje consistirá en la conexión asociativa entre dos complejos de representaciones. En este punto surgen tres cuestiones de fundamental importancia, a las cuales pretendemos dar respuesta en este artículo:

1. cómo se establece dicha conexión entre representación-palabra y representación-cosa,

2. cómo se descomponen esos complejos para dar lugar a un lenguaje articulado, y

3. qué rol juega la cosa del mundo, si es que alguno.

Palabras clave: Proyecto de Psicología Representación-cosa - Representación-palabra

\section{Cómo citar este artículo:}

Caorsi, C. (2015). Algunas observaciones sobre la concepción del lenguaje subyacente en el "Proyecto de una psicología para neurólogos". Palavras. Revista de Epistemología, Metodología y Ética del Psicoanálisis, 1(1), 5-16. Recuperado de www.revistas.unlp.edu.ar/palavras 


\section{ALGUNAS OBSERVACIONES SOBRE LA CONCEPCIÓN DEL LENGUAJE SUBYACENTE EN EL "PROYECTO DE UNA PSICOLOGÍA PARA NEURÓLOGOS”}

\section{Carlos Caorsi *}

La teoria del lenguaje esbozada en el "Proyecto de Psicología", se articula sobre la distinción entre dos tipos de representaciones: la representación-cosa y la representación-palabra. Ambas representaciones, que son en principio registros a nivel psíquico de influjos procedentes de estimulaciones, tienen su origen en la percepción sensorial (Freud, [1915] 2004:198-99). Y ambas, a su vez, tienen carácter complejo, es decir, son complejos asociativos de representaciones acústicas, visuales, kinestésicas, etc. (ibid.: 207). Lo que en principio parece distinguir unas de otras es que en tanto las representaciones-cosa son primariamente visuales -aunque contienen elementos acústicos, táctiles, kinestésicos-, las representacionespalabra son primariamente acústicas, pese a contener también elementos visuales y kinestésicos. Dice Freud de estas últimas en su trabajo sobre las afasias: "Suelen citarse cuatro ingredientes de la representación-palabra: la 'imagen sonora', la imagen visual 'de letras', la imagen motriz 'del lenguaje' y la imagen motriz 'de la escritura"' (ibid.: 207: 202). Como queda claro por esta cita, lo que Freud considera representación-palabra se limita a los aspectos materiales del signo, como ristras de fonemas o grafos, y no incluye el significado que hace de esa ristra de fonemas una palabra. De modo que la representaciónpalabra contiene sólo una parte de lo que habitualmente consideramos una palabra. La otra parte, su significado, será provisto por la conexión de la representación-palabra con la representación-cosa. En la monografia citada, Freud dice: "La palabra cobra su significado por su enlace con la representación-cosa, al menos si consideramos solamente

\footnotetext{
* Instituto de Filosofia, Facultad de Humanidades y Ciencias de la Educación de la Universidad de la República, Uruguay. Mail: cecaorsi@hotmail.com
} 
los sustantivos" (ibid.: 212). Pero, ¿'en qué consiste esta representacióncosa? Según Freud, en explícita referencia a John Stuart Mill, la filosofia nos enseña que la representación-cosa no contiene más que las mencionadas representaciones sensoriales y que "la apariencia de ser una "cosa" (Ding) a favor de cuyas diversas "propiedades" aboga cada impresión sensorial, surge sólo por el hecho de que a raíz del recuento de las impresiones sensoriales que hemos recibido de un objeto del mundo admitimos todavía la posibilidad de una serie mayor de nuevas impresiones dentro de la misma cadena asociativa" (ibíd.: 212). De acuerdo con esto, la representación-cosa es un complejo de sense data, y es este complejo el que al asociarse con la representación-palabra le confiere un significado.

De acuerdo con lo que llevamos visto tenemos dos complejos de sense data, a saber, la representación-cosa y la representación-palabra, y será la conexión que se dé entre ambos lo que atribuirá significado a este último. Luego, la relación semántica fundamental que dará lugar al desarrollo del lenguaje consistirá en la conexión asociativa entre dos complejos de representaciones. En este punto surgen tres cuestiones de fundamental importancia:

1. cómo se establece esa conexión,

2. cómo se descomponen esos complejos para dar lugar a un lenguaje articulado, y

3. qué rol juega la cosa del mundo, si es que alguno.

\section{1.}

La respuesta a la primera cuestión nos obliga a hacer un recorrido por la concepción del aparato psíquico desarrollado en el Proyecto. Trataremos que ese recorrido sea breve, aunque con ello podamos caer en algunas imprecisiones.

Freud concibe el aparato psíquico como un sistema de neuronas que puede estar en actividad o en reposo en virtud de que una cierta energía $Q$ transite o no por ellas. Este sistema está regido por el principio de inercia 
neuronal que enuncia que las neuronas procuran aliviarse de la cantidad (Freud, [1895] 2004: 340). De modo que siempre que se dé un incremento de Q, el sistema procurará eliminar esa cantidad para volver a su estado de reposo inicial. En tanto que el incremento de $Q$ será vivido a nivel del sistema como displacer, su eliminación procurará una vivencia de placer. Ahora bien, dicho sistema estará expuesto a estímulos provenientes de dos fuentes diferentes, por un lado recibirá estímulos del mundo exterior y por otro estímulos endógenos provenientes del propio organismo (estímulos pulsionales, hambre, deseo sexual, etc.). Los estímulos provenientes del exterior actuarán sobre un tipo especializado de neuronas, las neuronas $\varphi$ que Freud denomina pasaderas, ya que una vez trasmitido el estímulo vuelven a su estado inicial. Este tipo de neuronas son las que dan lugar a la percepción y, a los efectos de cumplir esa función, no deben ser modificadas por el estímulo que incide sobre ellas. A su vez, estas neuronas trasmiten el estímulo hacia el interior del sistema donde podrá la experiencia quedar registrada en la memoria. Pero para que este registro sea posible, es necesario que las neuronas que reciben el estimulo desde $\varphi$ resulten, a diferencia de aquellas, alteradas por la recepción del mismo. Así encontraremos en el interior del sistema un tipo diferente de neuronas, de carácter impasadero, que Freud denomina como neuronas $\psi$. Estas neuronas tienen lo que Freud llama barreras-contacto, que impiden trasmitir la Qn (cantidad de trasmisión intercelular) recibida hasta que no supere un determinado umbral. Una vez que el mismo es superado y se da la trasmisión de Qn, la vía por la cual la misma se realizó queda facilitada, es decir esa vía queda más sensible a la trasmisión. A tales efectos cabe señalar que las neuronas están interconectadas en redes y cada neurona $\psi$, tiene varias vías de trasmisión hacía otras neuronas $\psi$ del sistema. La facilitación se produce en la barrera contacto de una de esas vías de trasmisión y no en

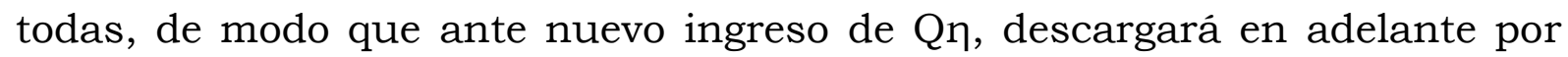
esa vía y no por otra de sus vías de trasmisión posible. Así se genera un camino de trasmisión, lo que Freud denomina una huella mnémica. Aún necesitamos una consideración más a los efectos de lograr un esclarecimiento del modo en que se establece la conexión. Cuando una 
neurona está cargada con Qn, decimos que esta investida. Bien, según vimos la descarga de Qn por una de las vías de conexión neuronal, crea una facilitación y esa facilitación genera que la descarga se dé en adelante por esa vía. Sin embargo es posible que el sistema desvie la vía de descarga aún en este caso. Ello se logra mediante la investidura de una neurona colateral. Supongamos que la neurona $a$, tiene vías de descarga hacia las neuronas $b$, c y $d$. Y supongamos además que se encuentra facilitada la vía de conexión de $a$ con $b$, de modo tal que siempre que $a$ es investida descargara hacia $b$. Pero supongamos además que la neurona $c$ es investida simultáneamente con la neurona $a$. En ese caso la descarga podría desviarse hacia $c$ y no realizarse a nivel de $b$, como lo suponía la facilitación anterior. Con ello se ha logrado incidir sobre los caminos de descarga establecidos por las huellas mnémicas. Ahora bien, esto obliga a introducir un nuevo actor en el sistema, que sería el encargado, en este caso, de investir la neurona $c$ colateral a los efectos de modificar el camino de descarga. Este actor es el Yo. El Yo debe ser concebido como un complejo de neuronas $\psi$, que retiene un cierto monto de investidura a los efectos de poder incidir sobre los caminos de descarga establecidos. Por medio de esta investidura podrá el Yo investir la neurona $c$ del ejemplo anterior y modificar el camino de descarga. Por el momento esto parece suficiente para le elucidación que nos proponemos.

Volvamos entonces a nuestro asunto; como señalamos, el sistema $\Psi$ recibe estímulos desde $\varphi$ y desde el polo pulsional. En el caso de los estímulos provenientes de $\varphi$ el sistema puede usar esa misma investidura para huir del estímulo mediante descarga por el sistema motor y volver a su estado de reposo. Se trata simplemente de alejarse del estímulo para eliminar su influjo. Pero si el estímulo es de carácter endógeno, como sucede con los estímulos pulsionales, el camino de huida no es un camino posible. El sistema no puede poner distancia con el estímulo ya que este se da en él mismo. Si sentimos hambre, no hay desplazamiento que podamos hacer, como no sea ir hacia el refrigerador, para librarnos del estímulo. Debemos realizar una acción, en este caso la ingesta de alimentos, que elimine el estímulo, y no huir de él. Esta acción que tiene como resultado la 
eliminación del estímulo es lo que Freud llama la acción específica. Veamos a modo de ejemplo la descripción debida a Freud de lo que denomina la primera experiencia de satisfacción (Ibid. 362). El niño siente hambre (estímulo de carácter endógeno) lo cual se manifiesta como una elevación de Qn que es vivida como displacentera. La primera reacción del niño es el llanto y el berreo, forma de descarga que no elimina el estímulo. Un adulto, la madre advierte la situación del niño y le provee alimento. Así, el auxilio de la madre permite que se realice la acción específica, lo cual elimina el estímulo displacentero. Aquí tenemos un estímulo endógeno vivido como displacer, seguido de un sentimiento de inervación corporal y de un sonido, seguido de la percepción de la madre. Ahora se ha generado un complejo asociativo constituido por representaciones correspondientes a la sensación de hambre, de la sensación kinestésica de la descarga motriz, de la sensación auditiva del llanto, de la sensación visual de la madre, etc. Una vez dado este conjunto de sensaciones en forma sucesiva o simultánea, se produce una facilitación entre las mismas que crea una huella mnémica. Dentro de este complejo asociativo tenemos una representación-cosa, los estímulos originados en la madre, primordialmente el visual y una representación sonora del llanto del niño. Si bien estamos lejos, en este último caso de una representación-palabra, sí estamos en los inicios de la génesis de la misma. Porque obsérvese que el llanto del niño, que no fue otra cosa que una descarga sobre el camino de la alteración interior, cobra en esta situación una función secundaria, a saber, la de advertir a la madre del estado del niño. Esta advertencia, que en esta ocasión solo lo es desde la perspectiva de la madre, ya contiene el germen de lo que será el uso del sonido por parte del niño como un modo de llamar a la madre. Una vez que el niño advierte que la madre responde a un sonido y aprende a generar ese sonido y no otro, habrá obtenido una representación-palabra, con su contenido acústico y kinestésico. Y el significado que esa representaciónpalabra será la representación-cosa constituida por la representación visual, etc. de la madre. De acuerdo con esto, parece que el modo fundamental en que se produce la asociación entre la representación-cosa y la 
representación-palabra es por la investidura simultánea de los dos complejos asociativos, lo cual crea una facilitación entre ellos.

\section{2.}

Vayamos ahora a nuestra segunda cuestión: ¿Cómo se descomponen esos complejos para dar lugar a un lenguaje articulado? Es importante señalar que aquí Freud se aboca a cómo se disciernen las partes dentro del complejo representación-cosa y no del correspondiente a la representaciónpalabra. ${ }^{1}$ Aquí debemos volver a la primera experiencia de satisfacción. Como vimos, allí se había producido un complejo asociativo entre: estímulo displacentero del hambre+representación-cosa, madre+representación de la descarga motora, succión. Supongamos ahora que vuelve a aparecer el estímulo del hambre; dada la facilitación que las experiencias anteriores establecieron entre las distintas representaciones, la vía de descarga irá hacia la representación de la madre y una vez investida ésta se dará camino a la descarga motora; succión. Pero supongamos que en esta instancia la madre no está presente. La sobreinvestidura de la representación-cosa madre, dará lugar a la alucinación de ésta y con ello se procederá a la descarga correspondiente. Pero como la madre no está presente, la acción específica no se llevará a cabo y la eliminación del estímulo displacentero tampoco. Para que se lleve a cabo la acción específica de un modo adecuado, será necesario esperar a la presencia real de la madre para iniciar la descarga motora. Pero por presencia real de la madre queremos decir la experiencia perceptiva de la misma. De modo que deben darse, por un lado la imagen percepción de la madre, es decir la representación-cosa generada por esa percepción, por otro la imagen de la madre contenida en la representación-cosa de la experiencia anterior, y se debe ver que hay una identidad entre las mismas de modo de dar lugar a la descarga motora. Pero

\footnotetext{
1 Aunque podría pensarse que la descomposición del complejo de representación palabra se da vía distinción sujeto/predicado, en concordancia con la descomposición de la representación-cosa en cosa y atributo. Desarrollaremos esto más adelante.
} 
el punto es que ambas representaciones-cosa no tienen que coincidir en todos sus componentes. En el interin la madre puede haberse cansado del Chanel $N^{\circ} 5$ y haber empezado a usar Poison, con lo cual ambos complejos representacionales van a ser diferentes en algunos de sus componentes. A esto se refiere Freud cuando señala que la representación-cosa, a diferencia de la representación-palabra, nos aparece como algo abierto, es decir que al complejo de representación-cosa pueden integrarse elementos nuevos (Freud, [1915] 2004: 212). O por tomar un ejemplo del propio Freud, mientras que en el recuerdo el pecho materno puede presentarse de frente con el pezón a la vista, en la percepción puede aparecer de perfil y con el pezón oculto. En este caso, dice Freud, el niño podrá asociar con huellas de acciones anteriores, como haber movido la cabeza y pasar a ver el pecho de perfil, para ahora realizar la acción inversa y tener el pecho de frente. Pero lo que nos interesa destacar aquí es la realización del discernimiento dentro de ambos complejos asociativos para lograr la identidad de percepción que daría lugar a la acción específica. Para ello debe retardar la descarga hasta haber logrado la referida identidad. Como resultado de ese discernimiento hallará algo constante, la cosa del mundo y algo variable, sus atributos. $O$ sea que el discernimiento del complejo se obtendrá por comparación con otros complejos percepción y será a partir de esa diferencia que se establezca la distinción cosa/atributo.

\section{3.}

Nos queda ahora el tercer punto, a saber, cuál podría ser el rol de la cosa en el mundo. Como acabamos de ver, la cosa del mundo resulta identificada por la identidad de percepción y esa identidad confirmada por el éxito de la acción específica. En este éxito la cosa se revela como independiente del sujeto y de los sense data, representación-cosa correspondiente, que pueda producir en éste. Dicha independencia se manifiesta en la diferencia entre la acción específica y la satisfacción alucinatoria de deseos. Así nos encontramos con que la cosa, que permite configurar el significado o la referencia de la palabra, por su asociación con 
la representación-palabra, vía representación-cosa, se constituye en un núcleo semántico independiente del sujeto. Por supuesto que dicho núcleo semántico, constituido por la cosa del mundo resulta en última instancia sólo accesible a través de sus atributos, es decir por las correspondientes representaciones-cosa. No accedemos a la cosa sino a través de las representaciones-cosa, especie de cualia resultantes en gran parte de nuestro sistema perceptivo; y es posible que distintos sujetos identifiquen la misma cosa a partir de diferentes atributos. Pero el discernimiento que conduce a la identidad de percepción, permite eliminar algunos de esos atributos como modo de identificación de la cosa. Por cierto, no es posible eliminarlos todos, ya que en ese caso no tendriamos acceso a la cosa, al carecer de representaciones-cosa; pero es necesario identificar algunos que deben presentarse como garantía de que la cosa está presente. Y esos atributos deben ser tales que identifiquen univocamente a la cosa. Es decir, la cosa es el referente de una descripción definida del tipo "el $x$, tal que $F x$ " donde $F$ es un conjunto de atributos tal que caracteriza a esa cosa univocamente. Así, por ejemplo, "El autor de 'Inhibición, sintoma y angustia' es Sigmund Freud", porque él escribió esa obra y nadie más lo hizo. Claro que esta no es la única descripción que identifica unívocamente a S. Freud, también lo hacen, por ejemplo, "el autor de 'La interpretación de los sueños", y "el fundador del psicoanálisis". De este modo tenemos más de una descripción que identifica unívocamente una misma cosa y de acuerdo con ello distintas personas podrian identificar la misma cosa mediante distintos atributos. Así alguien puede saber que Freud escribió 'La interpretación de los sueños', pero ignorar que escribió 'Inhibición, síntoma y angustia', de modo que identificará a Freud como el autor de la primera obra pero no con el autor de la segunda. De este modo, la representación cosa, puede ser accesible por distintos sujeto de modos diferentes, por medio de representaciones cosa diferentes. Esto sin embargo no genera problemas y es consistente con el modo en que Freud trata el problema de la identidad de percepción. A lo sumo podría tener como consecuencia que el sujeto no realizara, o pospusiera, la acción específica en una ocasión en que podría 
hacerlo. ${ }^{2}$ Lo que sí es importante y juega un rol crucial en la identidad de percepción es que la descripción que el sujeto asocia con la representacióncosa caracterice unívocamente a esta, porque de no hacerlo la realización de la acción específica no podría llevarse a cabo. Con ello hemos llegado a identificar el rol que juega la cosa del mundo. Veamos ahora las características de la misma que hemos logrado discernir:

a. En primer lugar, dicha "cosa" debe ser independiente del sujeto. Esta independencia es lo que permite explicar la diferencia entre la exitosa acción específica y la fracasada satisfacción alucinatoria de deseos. La diferencia entre ambas reside en que en el primer caso la cosa está presente junto con su representación-cosa y en el segundo sólo está presente la representación-cosa.

b. Esta diferencia nos permite reconocer la cosa como diferente de su representación, sea esta del tipo que fuere. Es decir, la cosa no es una representación. Si basándonos en el carácter abierto mencionado de la representación-cosa, tratáramos de identificar a esta con el conjunto de representaciones que deben coincidir para que se dé la identidad de percepción, no tendríamos modo de establecer la distinción señalada en a. Porque en la satisfacción alucinatoria también la identidad de percepción está presente; la representación-cosa alucinada coincide con la representación-cosa recordada de una satisfacción anterior; en otras cosas porque tal vez se trate de la investidura de esa misma representación.

c. Así, en el complejo asociativo que representa una cosa (su representación cosa) hay elementos que podríamos llamar circunstanciales (el aroma a Chanel $N^{\circ} 5$ ) y elementos constantes que aseguran la identidad para el sujeto que realiza la discriminación a los efectos de dar lugar a la descarga.

\footnotetext{
2 Véase el ejemplo de Freud que mencionamos más arriba a propósito de la percepción del pecho materno.
} 
Como resulta claro que no tenemos acceso a las cosas del mundo, sino a través de nuestras impresiones sensoriales, accedemos a la cosa a través de su representación. $Y$ en esa representación serán los elementos constantes los que nos permitan reconocer la identidad de la cosa. Es decir, accedemos a las cosas a través de sus atributos los cuales se expresan como elementos del complejo asociativo que constituye su representación cosa. Y en esta distinción entre la cosa y sus atributos, ya encontramos el germen de la distinción sujeto-predicado. Y esta distinción sintáctica surge del modo en que se descompone la representación-cosa. Cada uno de los componentes de la representación-cosa es un predicado que tomado conjuntamente con el resto de los componentes (predicados) del mismo complejo asociativo, permiten identificar a la cosa.

Así, los elementos analizados de la representación-cosa constituyen la semántica de las representaciones-palabra que las sobre-invisten. Un sustantivo como "Mamá" se asocia (adquiere su significado) con un complejo asociativo (representación-cosa) por medio del cual el niño identifica a su madre. Ese complejo asociativo contendrá elementos visuales, táctiles y de otro tipo, que son los rasgos que, tomados en su conjunto, permiten al niño reconocer a su madre y que darán lugar a adjetivos como, morena, rubia, suave, etc. Supongamos que la representación que el niño asocia con su madre está constituida por las sensaciones $\mathrm{s}_{1}, \mathrm{~s}_{2}, \ldots, \mathrm{s}_{\mathrm{n}}$. Cada una de esas $\mathrm{s}_{\mathrm{i}}$ es un rasgo o atributo de la madre y se expresa en un predicado: $\mathrm{p}_{1}, \mathrm{p}_{2}, \ldots$, $\mathrm{p}_{\mathrm{n}}$. Así podríamos describir a la madre como: la $x$ tal que $x$ es $\mathrm{p}_{1}$ y $x$ es $\mathrm{p}_{2}, \ldots$ y $x$ es $\mathrm{p}_{\mathrm{n}}$. Pero ninguno de los $\mathrm{p}_{\mathrm{i}}$ que componen dicho complejo descriptivo es una representación de la madre; ${ }^{3}$ la representación de la madre es la del complejo todo.

Para concluir. La semántica del 'Proyecto...' se origina en la representación cosa a partir de la cual las palabras adquieren sus significados. Con ello debemos identificar el origen de la semántica en este

\footnotetext{
3 Lo cual es una consecuencia de que no tenemos acceso a la cosa, sino a través de sus atributos, los cuales se expresan en componentes del complejo asociativo.
} 
tipo de representación. Por otro lado es a partir del modo en que la representación-cosa se articula que el lenguaje adquiere su propia articulación. Así la distinción sustantivo-adjetivo, tiene su fuente en los elementos constantes y variables de la representación-cosa, y en el proceso de discriminación que conduce a la identidad de percepción. Con ello la sintaxis de la representación-palabra parece tener su origen también en la representación-cosa. Por último esta representación-cosa, tiene a su vez su origen en la cosa del mundo, y es el modo en que esta última se manifiesta al sujeto. Por esta vía, el referente de la representación palabra trasciende la representación cosa y alcanza la cosa misma del mundo. Si adoptáramos la distinción fregeana entre sentido y denotación (Frege, [1892] 1973), podriamos forzar una analogía entra la representación-cosa freudiana y el sentido fregeano, en tanto que este contiene el modo de presentación de la referencia, y la referencia fregeana con la cosa freudiana.

\section{Referencias bibliográficas}

Frege, G. ([1892] 1973). "Sobre el sentido y la denotación", en Simpson, Thomas Moro, Semántica Filosófica, problemas y discusiones. Págs. 327. Buenos Aires: Siglo XXI.

Freud, S. ([1895] 2004). "Proyecto de psicología", en Obras Completas, tomo I. Buenos Aires: Amorrortu.

Freud, S. ([1915] 2004). "Lo inconciente", en Obras Completas, tomo XIV. Buenos Aires: Amorrortu. 